\title{
Review: childhood sexual abuse is associated with bulimia nervosa especially when there is psychiatric comorbidity
}

\author{
Wonderlich SA, Brewerton TD, Jocic Z, et al. Relationship of childhood sexual abuse and eating disorders. J Am Acad Child Adolesc \\ Psychiatry 1997 Aug;36:1107-15.
}

\section{Objective}

To determine the extent, nature, and specificity of any association between childhood sexual abuse and eating disorders by systematically reviewing the literature.

\section{Data sources}

Studies were identified by searching Medline and PsychLIT (1987-94 inclusive) using the index words child abuse, sexual child abuse, anorexia, bulimia, and eating disorders; by scanning the bibliographies of relevant papers; and by contacting researchers in the field.

\section{Study selection}

Relevant studies were selected if there were $\geqslant 10$ participants per group; eating disorders were assessed by interview using the Diagnostic and Statistical Manual of Mental Disorders, 3rd edition (DSM-III) or DSM-III-R or using valid and reliable questionnaires; and sexual abuse before age 18 years was assessed by interview or using appropriate questionnaires. Studies were excluded if there was major potential bias or inadequate statistical analysis for the research question.

\section{Data extraction}

2 groups of raters independently reviewed each study to determine if there was empirical support for specific hypotheses regarding the relation between childhood sexual abuse and eating disorders. Disagreements about whether a study spported a particular hypothesis were resolved through consensus agreement. A formal meta-analysis was not done.

\section{Main results}

53 studies met the selection criteria but many were judged to be inadequate and only some were relevant to each research question. 6 of 8 adequate studies of community samples supported the hypothesis that childhood sexual abuse was associated with bulimia nervosa. Other study designs produced contradictory results. Bulimia nervosa showed a greater association with abuse than did anorexia nervosa in 4 of the 6 studies with relevant data. All 7 adequate studies attempting to determine if child sexual abuse was specifically associated with eating disorders compared with other disorders found no such specific relation. 5 of 6 adequate studies found an association between abuse and greater psychiatric comorbidity in patients with eating disorders. The 3 adequate and relevant studies failed to find an association between the severity of eating disorder symptoms and childhood sexual abuse. 4 studies found an association between the magnitude of eating disturbances and particular features of childhood history in abused women such as decreased social competence, poor maternal relationship, and abuse severity. However, there was no consistency of findings among these studies.

\section{Conclusions}

Childhood sexual abuse is a non-specific correlate of bulimia nervosa. Such abuse is associated with greater psychiatric comorbidity but not with the overall severity of the eating disorder.

\section{Source of funding: no external funding.}

For article reprint: Dr S A Wonderlich, Department of Neuroscience, University of North Dakota School of Medicine and Health Sciences, 1919 North Elm Street North, Fargo, ND 58102, USA. Fax +1 7012934109 .

\section{Commentary}

The speculation about whether there may be a positive association between sexual abuse in childhood and the later development of clinical eating disorder has been widely considered over the past 15 years. It has sometimes been accepted uncritically as established truth. It is certainly plausible. After all, bulimia and anorexia nervosa differentially affect girls and seem to involve complex feelings about the body which might well have originated in adverse early sexual experience. Furthermore, many patients disclose such experiences in the clinic. The evidence from early research studies, however, does not consistently support all of the clinical speculation. More than one review reached broadly negative conclusions. ${ }^{12}$ The study by Wonderlich et al systematically re-examines publications up to the end of 1994

The authors of this review had to grapple with studies employing a wide variety of methods and samples. They used predetermined criteria of quality but not meta-analysis to sort out which studies could contribute to their conclusions. They were appropriately strict in applying their criteria.

This review supports the position of clinicians who consider that a history of childhood sexual abuse is worth seeking and may be an important consideration in their patients with eating disorders, but no more important than in many other patients. It may be especially relevant for those who have bulimia nervosa with comorbidity. Patients with such complex problems require careful thought. Finding a background of sexual abuse may prove to be important but should not lead to the conclusion that "all is now explained". Furthermore, most studies show that only a minority of patients with eating disorders report abuse and inferring past abuse from the fact of present eating disorder is unjustified.

$$
\begin{array}{r}
\text { Robert L Palmer, MB, BS } \\
\text { University of Leicester } \\
\text { Leicester, UK }
\end{array}
$$

1 Connor ME, Morse W. Sexual abuse and eating disorders: a review. Int I Eating Disord 1993;13:1-11.

2 Pope HG, Hudson JI. Is childhood sexual abuse a risk factor for bulimia nervosa? Am J Psychiatry 1992;149:455-63. 